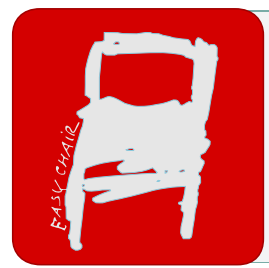

EPiC Series in Health Sciences

Volume 4, 2020, Pages 220-224

CAOS 2020. The 20th Annual Meeting of the International Society for Computer Assisted Orthopaedic Surgery

\title{
Optimal Targeting Display for Navigated Pelvic Screw Insertions
}

\author{
Prashant U. Pandey, Pierre Guy, Kelly A. Lefaivre, and Antony J. Hodgson \\ University of British Columbia, Vancouver, BC, Canada \\ prashant@ece.ubc.ca
}

\section{Introduction}

Surgical navigation can be used for complex orthopaedic procedures, such as iliosacral screw fixations, to achieve accurate and efficient results [11]. Although there have been studies studying the impact of navigation systems on surgical outcomes [6,3], we are not aware of any studies that have quantified the effect of how information regarding the surgical navigation scene is displayed to surgeons on conventional monitors. However, the display of information can have a measurable effect on both accuracy and time required to perform the navigated surgery, as the surgical scene can be presented in many different formats [9]. Optimizing surgical accuracy potentially helps improves patient safety by reducing screw malplacement [11], while optimizing time efficiency reduces opportunity cost [1]. Therefore, we designed a study to determine the optimal visualizations for performing navigated pelvic screw insertions. The findings of this study can be used to more systematically design visualization components of a navigation system.

\section{Methods}

We conducted a user study to investigate which visualization techniques helped users effectively navigate a surgical tool to a screw target - a core task during pelvic fracture fixations. Our study simulated the procedure using a dry pelvis model and optical tracking. We proposed a series of $2 \mathrm{D}$ and $3 \mathrm{D}$ visualizations with varying representations of the screw targets and the guide tool, and measured the accuracy and time required by each participant to perform the task. We acquired a CT scan of a radio-opaque pelvis bone model (Sawbones, WA, USA), and placed 10 virtual cylindrical targets (diameter: $7.3 \mathrm{~mm}$ and varying lengths) within the CT image using the open-source Slicer software [2]. To simulate a navigated surgery, we tracked the pelvis model and a surgical wire guide tool using an NDI Vega and rigid body markers (NDI, ON, Canada). We calibrated the wire guide to define its tip and axis relative to the attached marker [5], and subsequently performed point-based registration to align the pelvis to its CT image.

We designed one 3D and five 2D visualizations to represent the surgical scene, using Slicer and SlicerIGT [10]. The 2D views were 1) a 'bullseye' view looking into the screw channel, 
oriented such that the anatomical axes on the display were aligned with the physical pelvis model, 2) a 'rotated bullseye' view such that its orientation was rotated $90^{\circ}$ clockwise with respect to the physical pelvis, 3) a 'target-fixed' view showing the target cross-sections along the screw axes in two orthogonal planes nominally aligned with axial and coronal planes, 4) a 'tool-fixed (translation)' view which fixed the tool-tip's position in the centre of the view such that the image translated around it during tool motion, shown from an axial and coronal plane, and 5) a 'tool-fixed (translation and rotation)' view which fixed the tool-tip's position and orientation in the center of the view such that the image translated and rotated around it during tool motion. The 'bullseye' and 'tool-fixed (translation and rotation)' views were chosen to mimic commonly used views in commercial and open-source navigation frameworks, while the others were designed to test how changes in perspective and relative motion affect navigation performance. The 3D view displayed two volumetric renderings of the CT scan approximately representing the perspective of the user looking at the physical pelvis model. Each rendering was cropped by orthogonal planes defined by the tool's pose aligned with the tool's trajectory (Figure 1).

We asked each participant to navigate the tool to five targets for each of the visualizations, using only the information presented on a 40 inch monitor (Samsung, Model: LN40D630M3F, South Korea) placed directly in front of the participant. The pelvis model was also covered with an opaque cloth so participants could not use direct visual features from the model to assist in targeting. The order of visualizations was randomized for each participant. For each target, we recorded the tool trajectory relative to the target entry. We asked each user which visualization they preferred most - with the exception of the two bullseye views. We combined this preferred view with a bullseye, and asked them to navigate again to 5 random targets. In total, 35 trajectories were acquired per participant. We measured the total time required to complete navigation, the time to reach a threshold accuracy (within $3.65 \mathrm{~mm}$ of target entry and $5^{\circ}$ of the target axis), and the distance and the rotational errors at the end of the trajectory. We used a two-way analysis of variance and the Tukey-Kramer test to infer statistical differences between visualizations $(p<0.05)$.

\section{Results}

We recruited 8 novice participants who had no experience performing surgical fixations and using navigation in surgery. 5 participants preferred the 'target-fixed' visualization, 2 the 'toolfixed (translation and rotation)' view, and 1 the 3D view (Table 1 ).

Overall, the standard bullseye view had the lowest mean required time for navigation $(26.9 \mathrm{~s})$ and this was statistically lower than the 'rotated bullseye', 'target-fixed' and 3D visualizations. The 'target-fixed and bullseye' combination had a mean time of $30.9 \mathrm{~s}$, which was statistically lower than the 3D view. The 'bullseye' and 'target-fixed + bullseye' views also had the lowest mean time to threshold accuracy of $11.9 \mathrm{~s}$ and $13.3 \mathrm{~s}$ respectively, which were both statistically lower than the 'target-fixed', 'tool-fixed (translation)', and 3D views. All 2D views and combinations of bullseye with preferred views had statistically lower means for distance and rotational accuracies when compared to the $3 \mathrm{D}$ view alone, with no other statistical differences in accuracies. 

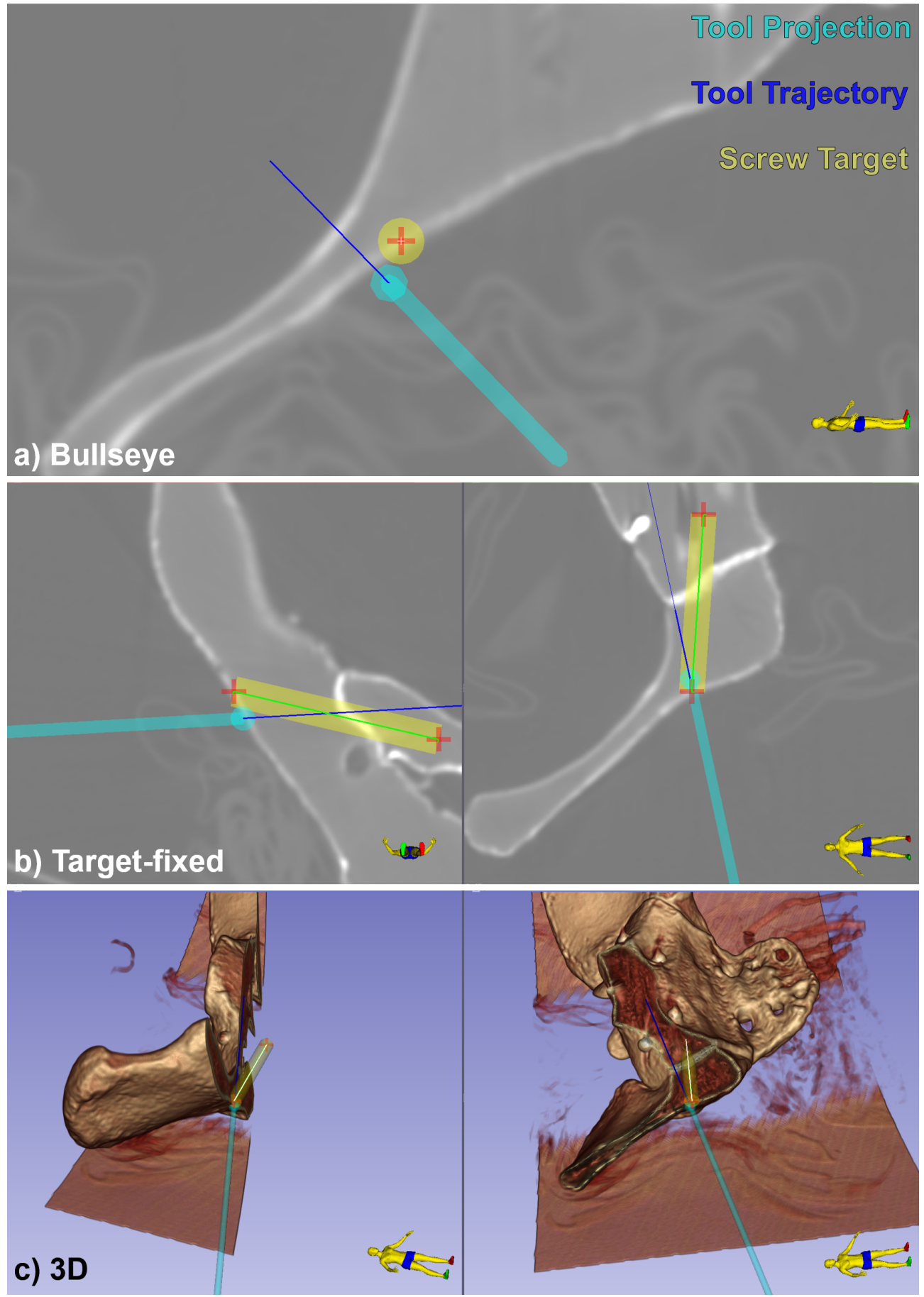

Figure 1: Three visualizations used in the study a) bullseye, b) target-fixed and c) 3D view. The other three visualizations differ only in orientation or in their dynamic representation during tool motion. 


\begin{tabular}{l|l|l|l|l}
\hline Visualization & $\begin{array}{l}\text { Total time } \\
(\mathbf{s})\end{array}$ & $\begin{array}{l}\text { Time to } \\
\mathbf{3 . 6 5 m m} \\
\text { and 5 } \mathbf{5}^{\circ}(\mathrm{s})\end{array}$ & $\begin{array}{l}\text { Distance } \\
\text { Accuracy } \\
(\mathbf{m m})\end{array}$ & $\begin{array}{l}\text { Rotational } \\
\text { Accuracy } \\
\left({ }^{\circ}\right)\end{array}$ \\
\hline Bullseye & $26.9(16.8)$ & $11.9(10.2)$ & $0.88(0.67)$ & $0.77(1.91)$ \\
\hline Rotated Bullseye & $51.9(34.0)$ & $22.3(14.4)$ & $1.05(0.54)$ & $0.47(0.24)$ \\
\hline Target-fixed & $53.3(54.1)$ & $\mathbf{2 7 . 9 ( 2 4 . 7 )}$ & $1.58(1.73)$ & $1.22(0.71)$ \\
\hline Tool-fixed (translation) & $49.7(23.9)$ & $\mathbf{2 9 . 2 ( 2 0 . 0 )}$ & $1.27(0.67)$ & $1.05(0.57)$ \\
\hline $\begin{array}{l}\text { Tool-fixed (translation }+ \\
\text { rotation) }\end{array}$ & $39.7(18.5)$ & $21.5(12.2)$ & $1.03(0.50)$ & $1.00(0.62)$ \\
\hline 3D & $\mathbf{6 1 . 2 ( 5 0 . 7 )}$ & $\mathbf{4 1 . 0 ( 3 0 . 9 )}$ & $\mathbf{4 . 2 2 ( 2 . 9 4 )}$ & $\mathbf{6 . 1 5}(4.73)$ \\
\hline Target-fixed + Bullseye & $30.9(22.6)$ & $\mathbf{1 3 . 3 ( 6 . 6 )}$ & $1.04(0.61)$ & $0.51(0.33)$ \\
\hline $\begin{array}{l}\text { Tool-fixed (translation }+ \\
\text { rotation)+ Bullseye }\end{array}$ & $31.0(15.3)$ & $15.8(4.0)$ & $1.37(0.76)$ & $0.41(0.40)$ \\
\hline 3D + Bullseye & $51.8(17.9)$ & $16.0(19.9)$ & $0.93(0.61)$ & $0.41(0.24)$ \\
\hline
\end{tabular}

Table 1: Means and standard deviations of each measure across all participants, grouped by visualization type. Green indicates the mean was statistically lower than at least one of the other visualizations within a column, and red indicates the mean was statistically higher than at least one of the other visualizations within a column.

\section{Discussion And Conclusion}

Our results indicate that the 'bullseye' and 'target-fixed + bullseye' views are the most effective visualizations for navigated screw fixation, given their statistically shorter times and higher accuracies than other visualizations. We also found that the alignment of the visualization with respect to the patient's physical orientation has a significant impact on navigation time, indicated by the statistically longer time for the 'rotated bullseye' view. Moreover, the 3D view was significantly worse than the other visualizations in all outcome measures, suggesting it is not an effective way to visualize navigation on its own. We believe this is because it is difficult to visually estimate the pose of the tool with respect to a target given only 3D representations. However, when combined with a bullseye, the $3 \mathrm{D}$ visualization had significantly improved displacement and rotational accuracy, albeit with only marginally reduced targeting time. This finding is supported in literature for visualizations and displays outside of the surgical context $[9,8]$. Our study did not directly assess navigation displays using alternative technologies such as head-mounted displays [7] or directly on the surgical instrument [4].

This study focused only on the task of tool navigation to the target, but a real procedure would involve assessing the planned screw position and drilling into tissue, for which navigation information might also be used. Furthermore, our study does not model the presence of tissue which a surgeon would have to navigate through in a real surgery. Overall we recommend that surgical navigation systems implement a 'bullseye' due to its effectiveness. However, it is also worth augmenting the 'bullseye' with a 'target-fixed' view given that performance is not affected and the additional information could add value during the surgical planning and drilling tasks.

\section{References}

[1] Abhishek Chatterjee, Michael J. Payette, Christopher P. Demas, and Samuel R.G. Finlayson. Opportunity cost: A systematic application to surgery. Surgery, 2009. 
[2] Andriy Fedorov, Reinhard Beichel, Jayashree Kalpathy-Cramer, Julien Finet, Jean Christophe Fillion-Robin, Sonia Pujol, Christian Bauer, Dominique Jennings, Fiona Fennessy, Milan Sonka, John Buatti, Stephen Aylward, James V. Miller, Steve Pieper, and Ron Kikinis. 3D Slicer as an image computing platform for the Quantitative Imaging Network. Magn. Reson. Imaging, 30(9):1323-1341, nov 2012.

[3] Florian Gras, Ivan Marintschev, Arne Wilharm, Kajetan Klos, Thomas Mückley, and Gunther O Hofmann. 2D-fluoroscopic navigated percutaneous screw fixation of pelvic ring injuries - a case series. BMC Musculoskelet. Disord., 11(1):153, dec 2010.

[4] Marc Herrlich, Parnian Tavakol, David Black, Dirk Wenig, Christian Rieder, Rainer Malaka, and Ron Kikinis. Instrument-mounted displays for reducing cognitive load during surgical navigation. Int. J. Comput. Assist. Radiol. Surg., 12(9):1599-1605, sep 2017.

[5] Burton Ma, Niloofar Banihaveb, Joy Choi, Elvis C. S. Chen, and Amber L. Simpson. Is posebased pivot calibration superior to sphere fitting? In Robert J. Webster and Baowei Fei, editors, Med. Imaging 2017 Image-Guided Proced. Robot. Interv. Model., volume 10135, page 101351U, mar 2017.

[6] Kuo Ti Peng, Yen Yao Li, Wei Hsiu Hsu, Meng Huang Wu, Jen Tsung Yang, Chu Hsiang Hsu, and Tsung Jen Huang. Intraoperative computed tomography with integrated navigation in percutaneous iliosacral screwing. Injury, 44(2):203-208, feb 2013.

[7] Praneeth Sadda, Ehsan Azimi, George Jallo, Jayfus Doswell, and Peter Kazanzides. Surgical navigation with a head-mounted tracking system and display. In Stud. Health Technol. Inform., volume 184, pages 363-369. IOS Press, 2013.

[8] H.S. Smallman, M. St. John, H.M. Oonk, and M.B. Cowen. Information availability in 2D and 3D displays. IEEE Comput. Graph. Appl., 21(4):51-57, 2001.

[9] M. Tory, A. E. Kirkpatrick, M. S. Atkins, and T. Moller. Visualization task performance with 2D, 3D, and combination displays. IEEE Trans. Vis. Comput. Graph., 12(1):2-13, jan 2006.

[10] Tamas Ungi, Andras Lasso, and Gabor Fichtinger. Open-source platforms for navigated imageguided interventions. Med. Image Anal., 33:181-186, oct 2016.

[11] Jörn Zwingmann, Oliver Hauschild, Gerrit Bode, Norbert P. Südkamp, and Hagen Schmal. Malposition and revision rates of different imaging modalities for percutaneous iliosacral screw fixation following pelvic fractures: A systematic review and meta-analysis. Arch. Orthop. Trauma Surg., 133(9):1257-1265, sep 2013. 